\title{
Avaliação da Temperatura de Transição Vítrea de Compósitos Poliméricos Reparados de Uso Aeronáutico
}

\author{
Jane M. F. de Paiva \\ Centro Técnico Aeroespacial, SP \\ Engenharia Ambiental, UNISAL, SP
}

Sérgio Mayer

EMBRAER, SP

ITA, SP

Geraldo M. Cândido, Mirabel C. Rezende
Centro Técnico Aeroespacial, SP

\begin{abstract}
Resumo: Este trabalho mostra a avaliação da temperatura de transição vítrea $\left(\mathrm{T}_{\mathrm{g}}\right)$, por DMTA, de três famílias de compósitos poliméricos reparados, tendo como laminados base tecidos de fibras de carbono/resina epóxi modificada com elastômero (F584), vidro/resina epóxi (F161) e aramida/resina epóxi (F161). Os compósitos foram laminados manualmente por processo convencional e curados em autoclave de indústria aeronáutica. Posteriormente, danos foram simulados sendo, em seguida, reparados adotando-se a técnica de sobreposição de camadas de pré-impregnados de resina epóxi (F155), com adição de filme adesivo de epóxi (FM 73). As curvas DMTA mostram os efeitos da combinação de diferentes sistemas de resinas na $T_{g}$ e, conseqüentemente, na temperatura de serviço do componente reparado. O material de reparo utilizado, baseado em préimpregnados com resina epóxi $\mathrm{F} 155$ e filme adesivo de epóxi, provocou a redução da $\mathrm{T}_{\mathrm{g}}$ dos compósitos reparados. Para os laminados base com tecido de fibras de carbono e vidro foram verificadas reduções de aproximadamente $30^{\circ} \mathrm{C}$ na $\mathrm{T}_{\mathrm{g}}$. Este efeito foi mais pronunciado (redução de aproximadamente $40^{\circ} \mathrm{C}$ ) quando o laminado reparado de aramida foi submetido a condicionamento higrotérmico a temperatura e umidade elevadas. Esta redução na $\mathrm{T}_{\mathrm{g}}$ dos laminados reparados é atribuída à migração do adesivo para o pré-impregnado de resina epóxi F155 utilizado no reparo, durante o processo de cura, e à plasticização do sistema polimérico pela água, durante o condicionamento higrotérmico. A redução da $\mathrm{T}_{\mathrm{g}}$ leva a uma conseqüente redução da temperatura de serviço do compósito polimérico reparado.
\end{abstract}

Palavras-Chave: Compósito polimérico, resina epóxi, reparo, $T_{g}$, DMTA.

\section{Evaluation of Glass Transition Temperature of the Repaired Polymeric Composites of Aeronautical Use}

Abstract: This work shows the evaluation of the glass transition temperature $\left(\mathrm{T}_{\mathrm{g}}\right)$ by DMTA of three different families of repaired polymeric composites, manufactured with carbon fiber fabric/epoxy F584, glass fabric/epoxy F161 and aramide fabric/epoxy F161, respectively. The composites were laminated by conventional hand lay-up process used in aeronautical industry. Afterwards, simulated damages, scarf type, were made and overlapping layers of F155 epoxy resin prepregs repaired them. The DMTA curves show the effects of combination of different systems resins on the $\mathrm{T}_{\mathrm{g}}$ values and, consequently, on the service temperature of the repaired laminate. The used repairing material (based on epoxy resin F155 and on epoxy adhesive film FM73) decreased the $\mathrm{T}_{\mathrm{g}}$ of the repaired laminates in approximately $30^{\circ} \mathrm{C}$ for the carbon and glass laminates. The conditioning of aramide laminate under elevated humidity and temperature provoked a more decrease of the $\mathrm{T}_{\mathrm{g}}$ (reduction of approximately $40{ }^{\circ} \mathrm{C}$ ). The $\mathrm{T}_{\mathrm{g}}$ reduction for the repaired laminates is attributed to the adhesive film migration into the F155 resin system during the cure process and also to the resin plasticization by water diffusion during the hygrothermal conditioning. The $\mathrm{T}_{\mathrm{g}}$ reduction leads to a decrease of the service temperature of the repaired polymeric composite.

Keywords: Polymeric composite, epoxy resin, repair, glass transition, DMTA.

\section{Introdução}

Os compósitos poliméricos de uso aeronáutico são materiais avançados de engenharia constituídos de fibras de reforço, como aramida, vidro ou carbono (na forma de fitas unidirecionais ou tecidos), combinadas com uma matriz polimérica, geralmente, de resinas epóxi, fenólica, bismaleimida ou poliimida ${ }^{[1-5]}$.

Particularmente, as resinas do tipo epóxi apresentam boas propriedades mecânicas, resistência química, baixa

Autor para correspondência: Mirabel C. Rezende, Divisão de Materiais - AMR/IAE/CTA, Praça Marechal do Ar Eduardo Gomes 50, Vila das Acácias, CEP: 12228-904, São José dos Campos, SP. E-mail: mirabel@iae.cta.br 
absorção de umidade, baixa porcentagem de contração e fácil processamento. As propriedades adequadas desse tipo de resina para o setor aeronáutico têm ampliado a sua aplicação no processamento de compósitos estruturais ${ }^{[3,6,7]}$.

Devido à crescente aceitação de tais materiais em aeronaves civis e militares está se tornando cada vez mais importante o reparo das peças e/ou estruturas manufaturadas com compósitos poliméricos. Por isto, na área aeronáutica vários trabalhos têm sido dedicados ao aprimoramento das técnicas de reparos, abordando diferentes metodologias, eficiência de diferentes materiais usados e a adequação de técnicas de re$\operatorname{paro}^{[8-12]}$.

Nos últimos anos, na indústria aeronáutica tornou-se comum a utilização de pré-impregnados ("prepreg") para a moldagem ou laminação dos compósitos poliméricos estruturais $^{[2,13,14]}$. O pré-impregnado consiste na combinação de uma matriz de resina polimérica com reforços de fibras (na forma de fitas unidirecionais ou tecidos) em um estágio semiacabado, podendo ser utilizado no processo da manufatura de componentes com diferentes geometrias ${ }^{[15]}$.

A utilização de pré-impregnados facilita a laminação dos compósitos de uso aeronáutico, pois esses são colocados esquematicamente sobre moldes metálicos, reduzindo-se o tempo de moldagem e podendo-se controlar a relação fibra/ resina, número de camadas, orientação do reforço, etc. Portanto, a utilização de pré-impregnados facilita muito a realização de reparos em aeronaves ${ }^{[10,11,15]}$.

Para que o compósito polimérico reparado atenda aos requisitos de serviço exigidos no setor aeronáutico, além das elevadas propriedades mecânicas (tração, compressão, cisalhamento, etc.), é importante que seja verificada a sua temperatura máxima de serviço, baseando-se no conhecimento da temperatura de transição vítrea $\left(\mathrm{T}_{\mathrm{g}}\right)$, que pode ser determinada por análise térmica dinâmico-mecânica (DMTA) [2,16-18]. Em geral, a temperatura de transição vítrea $\left(T_{\mathrm{g}}\right)$ está relacionada à região de transição vítrea para uma ampla variedade de polímeros termoplásticos e termorrígidos ${ }^{[19]}$.

Nos últimos anos, a técnica de DMTA também tem despertado o interesse para a caracterização de pré-impregnados e compósitos estruturais de matrizes poliméricas aplicadas no setor aeronáutico ${ }^{[2,14,20]}$. Uma das vantagens desta técnica é a possibilidade de determinação da $\mathrm{T}_{\mathrm{g}}$ diretamente em pequenas barras ou vigas do material a ser analisado ${ }^{[19,21]}$.

A técnica de DMTA apresenta sensibilidade de aproximadamente três ordens de grandeza superior à de uma técnica de análise térmica convencional como, por exemplo, DSC, TMA, etc. ${ }^{[22]}$. Esta característica é particularmente interessante para a determinação de $T_{g}$ em compósitos, pois devido aos vários componentes presentes neste tipo de material torna-se difícil ou imprecisa a determinação da sua $\mathrm{T}_{\mathrm{g}}$ utilizando-se, por exemplo, a técnica de calorimetria exploratória diferencial (DSC).

A transição vítrea ocorre quando as cadeias moleculares de um polímero adquirem energia suficiente (geralmente de fonte térmica) para superar as barreiras de energia necessárias à rotação de ligações. Sob estas condições o material passa do estado vítreo, caracterizado pela mobilidade limitada, para o estado de mobilidade, atingindo o equilíbrio termodinâmico. A diminuição do módulo de armazenamento (E') associado com esta mudança consiste de cerca de três ordens de grandeza para um polímero amorfo. A região de transição vítrea também é caracterizada por aumento no módulo de perda (E") e na tangente de perda $\left(\tan \delta=E^{\prime \prime} / E^{\prime}\right)^{[19,23,24]}$.

$\mathrm{Na}$ área de compósitos poliméricos, avaliando-se o módulo de perda (E”) ou tan delta também pode-se verificar mudanças de fatores microscópicos, tais como relaxações moleculares, ou fatores macroscópicos, como falha de adesão interfacial ${ }^{[21,24,25]}$.

Diante do exposto, no presente trabalho foi realizada a determinação da temperatura de transição vítrea, via DMTA, de compósitos laminados reparados de resina epóxi reforçados com fibras sintéticas (carbono, vidro e aramida).

A intenção deste trabalho foi analisar amostras de corposde-prova de compósitos reparados após estes terem sido previamente submetidos a ensaios de tração em temperatura elevada $\left(82 \pm 5^{\circ} \mathrm{C}\right)^{[10,11]}$, isto porque, durante os ensaios de tração, verificou-se que vários corpos-de-prova, principalmente após condicionamento higrotérmico (temperatura de $65 \pm 5^{\circ} \mathrm{C}$ e teor de umidade relativa de $95 \pm 5 \%$ ) até a saturação, estavam rompendo nas regiões do filme de epóxi (FM73) utilizado como adesivo no reparo ou na região da interface do reparo/laminado base e não no laminado base, sendo este último o esperado para reparos estruturais ${ }^{[10,11]}$. Portanto, a partir destas observações, realizou-se uma investigação minuciosa, utilizando-se a análise por DMTA, para determinar a temperatura de transição vítrea $\left(\mathrm{T}_{\mathrm{g}}\right)$ de todos os compósitos (sem reparo e reparados) e do filme adesivo utilizado.

\section{Experimental}

\section{Laminação dos compósitos, simulação dos danos e reparos}

Para este estudo, primeiramente, foram laminados três tipos de compósitos base através do empilhamento de seis camadas de pré-impregnados de tecido de fibras de carbono, vidro e aramida, respectivamente, com orientação $\left(0^{\circ} / 90^{\circ}\right)_{6}$, contendo resinas epóxi (códigos F584 e F161).

As resinas epóxi dos pré-impregnados ("prepregs") são do tipo multicomponentes e são baseadas no diglicidil éter de bisfenol-A, conhecido pela abreviação DGEBA (Diglycidyl Ether of Bisphenol A), com agentes de cura do tipo amina. A resina de código F584 também apresenta em sua formulação um modificador do tipo elastomérico. Os pré-impregnados utilizados foram fornecidos pela Hexcel Composites.

A cura dos laminados foi realizada em autoclave, atingindo a temperatura de $177^{\circ} \mathrm{C}$, pressão de aproximadamente 0,7 MPa e vácuo de aproximadamente $0,08 \mathrm{MPa}$.

Após a cura, os compósitos foram desmoldados e inspecionados por ultra-som (Reflectoscope S80, acoplado ao emissor Automation X19265, em freqüência de $5 \mathrm{MHz}$ ), de modo a detectar possíveis defeitos de fabricação (trincas, porosidades, delaminações, etc.), que pudessem comprometer a estrutura do laminado base. 


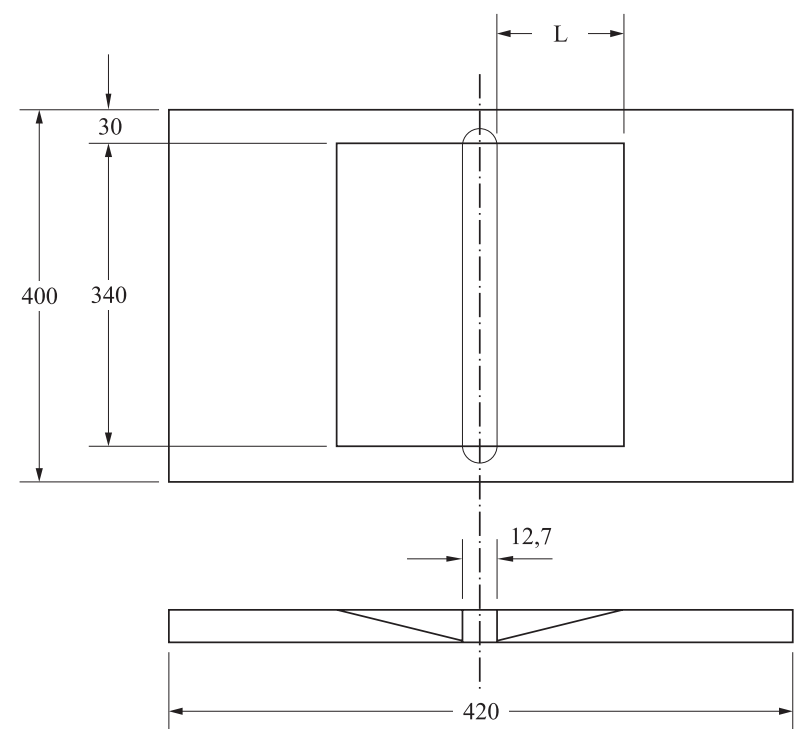

Figura 1. Representação esquemática mostrando o local da realização do chanfro, onde (L) corresponde a um dos lados do chanfro, e a dimensão de $12,7 \mathrm{~mm}$ corresponde ao rasgo produzido nos laminados base. Dimensões em $\mathrm{mm}$.

Em seguida, os laminados base foram cuidadosamente preparados para serem reparados. Para isto, estes foram usinados de acordo com a Figura 1, com o intuito de simular a ocorrência de danos. Esta operação simula o reparo em campo, ou seja, o reparo diretamente em peças que poderiam ter sofrido danos como, por exemplo, por impacto, compressão, etc.

Na seqüência, o dano posicionado no centro do laminado base (Figura 1) foi reparado com a utilização da técnica de chanfro para permitir o escalonamento das camadas, mantendo a geometria o mais próximo possível da original.

Para os reparos foram utilizadas oito camadas de pré-impregnados de resina epóxi F155/tecidos de fibras de carbono, vidro e aramida (prepregs fornecidos pela Hexcel Composites), dependendo do respectivo material de reforço do laminado base, e filme de adesivo epóxi (código FM73) com 0,045 psf (pound square foot), fornecido pela CytecFiberite, para auxiliar na adesão da interface reparo/laminado base.

Os laminados com os reparos foram levados novamente para a cura da resina em estufa a $121^{\circ} \mathrm{C}$ por $1 \mathrm{~h}$, sob vácuo de no mínimo 0,078 $\mathrm{MPa}$.

Para o reparo foi utilizado pré-impregnado com resina epóxi do tipo F155 porque o prepreg contendo este tipo de resina, atualmente, é o utilizado em maior escala nas oficinas de reparos da indústria nacional, devido a temperatura de cura ser de aproximadamente $121^{\circ} \mathrm{C}$, o que facilita a execução dos reparos em estufa ou até mesmo em campo. Utilizando o prepreg com resina epóxi de cura a $121{ }^{\circ} \mathrm{C}$ também se evita ampliar os danos, provocando delaminação, o que poderia ocorrer caso fosse utilizado prepreg contendo resina de temperatura de cura mais alta. Outro motivo para utilização da resina epóxi do tipo F155 está relacionado à diminuição do custo do reparo, pois o prepreg de resina epóxi F155 é de menor custo. A utilização de outro sistema de resina iria aumentar o custo e a quantidade de variáveis.
Após a cura dos laminados reparados foram cortados corpos-de-prova para ensaios de tração, os quais foram apresentados em trabalhos anteriores ${ }^{[10,11]}$. O corte dos corpos-de-prova foi realizado na posição transversal ao rasgo, sendo que bem no centro dos corpos-de-prova ficaram localizados os rasgos reparados com o filme adesivo epóxi.

Desta forma, os laminados reparados preparados foram:

- Laminado base de pré-impregnado de tecido de fibras de carbono/resina epóxi F584 reparado com pré-impregnado de tecido de fibras de carbono/resina epóxi F155.

- Laminado base de pré-impregnado de tecido de fibras de vidro/resina epóxi F161 reparado com pré-impregnado de tecido de fibras de vidro/resina epóxi F155.

- Laminado base de pré-impregnado de tecido de fibras de aramida/resina epóxi F161 reparado com pré-impregnado de tecido de fibras de aramida/resina epóxi F155.

\section{Análise microestrutural dos laminados base processados}

A qualidade dos laminados foi verificada por meio da análise microestrutural pelo uso de um microscópio óptico, marca Olympus (modelo B061) utilizando-se amostras (dimensão de aproximadamente: $15 \mathrm{~mm}$ x $10 \mathrm{~mm}$ x $3 \mathrm{~mm}$ ) cortadas dos laminados, e que foram previamente embutidas em resina fenólica e moldes de PVC, tendo as superfícies (superior e inferior da resina de embutimento) lixadas e polidas para avaliar a compactação das camadas e a distribuição das fibras e resina no interior dos laminados.

\section{Análise Térmica Dinâmico-Mecânica (DMTA)}

Para a realização das análises térmicas dinâmico-mecânicas foi utilizado um equipamento DMA, modelo 2980, da TA Instruments acoplado a um termoanalisador. As amostras de laminados reparados foram cortadas de corpos-deprova, previamente ensaiados em tração, nas dimensões de, aproximadamente, $64 \mathrm{~mm} \times 13 \mathrm{~mm} \times 3 \mathrm{~mm}$ e submetidas à solicitação mecânica tipo flexão, a $1 \mathrm{~Hz}$ de freqüência, amplitude de oscilação de $10 \mu \mathrm{m}$, razão de aquecimento $3^{\circ} \mathrm{C} \cdot \mathrm{min}^{-1}$ e intervalo de temperatura de 30 a $250{ }^{\circ} \mathrm{C}$.

Para o laminado base de tecido de fibras de carbono reparado foram analisadas três amostras, retiradas de diferentes regiões do corpo-de-prova de tração ensaiado. A primeira foi cortada no início do reparo próximo ao tab do lado da falha; a segunda próxima ao $t a b$, do lado oposto, e a terceira na região central do corpo-de-prova, conforme mostrado na Figura 2.

Para os outros laminados citados foram ensaiadas duas amostras de cada corpo-de-prova, retiradas respectivamente da região próxima à falha e da região central do corpo-deprova.

As amostras de laminados reparados de tecido de fibras de aramida foram analisadas por DMTA após serem submetidas a condicionamento higrotérmico (temperatura de $65 \pm 5^{\circ} \mathrm{C}$ e teor de umidade relativa de $95 \pm 5 \%$ ), para verificar porque praticamente todos os corpos-de-prova submetidos a estas condições estavam falhando no ensaio de tração sem atingir as propriedades mecânicas requeridas para o setor aeronáutico. 


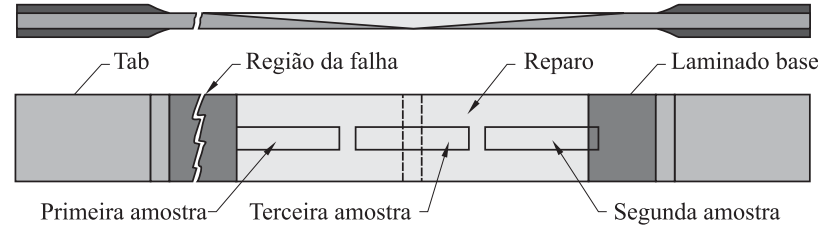

Figura 2. Representação esquemática do local de retirada de amostras dos corpos-de-prova de tração para análise por DMTA.

$\mathrm{O}$ condicionamento higrotérmico foi realizado de acordo com a norma ASTM D 5229/D5229 M-92. Os parâmetros foram programados em uma câmara de condicionamento higrotérmico modelo SSE-800M/MC, fabricada pela Equilam Ind. Com. Ltda.

Para avaliar a contribuição do filme adesivo epóxi e determinar sua $\mathrm{T}_{\mathrm{g}}$ isoladamente, foi realizada a cura deste adesivo em estufa, submetendo-o ao mesmo ciclo de cura dos laminados reparados e, na seqüência, realizada a análise do filme de epóxi curado por DMTA.

\section{Resultados e Discussão}

\section{Qualidade dos compósitos processados}

Os resultados obtidos por inspeção ultra-sônica dos laminados base foram considerados bons, pois os sinais de atenuação registrados no sistema de aquisição de dados, com unidades de níveis de voltagem, mostraram que não ocorreram descontinuidades do tipo vazio, bolha, trinca e delaminação nos compósitos. Estes resultados atestaram, portanto, que os laminados base processados estavam em conformidade com o padrão de qualidade exigido pelo setor aeronáutico, ou seja, sem defeitos na estrutura dos laminados, e poderiam ser utilizados para o estudo de reparo proposto. Os dados obtidos nesta fase do trabalho foram atribuídos à adequada metodologia de fabricação utilizada na indústria aeronáutica, a qual inclui procedimentos desde a armazenagem e manuseio dos pré-impregnados até o corte cuidadoso dos

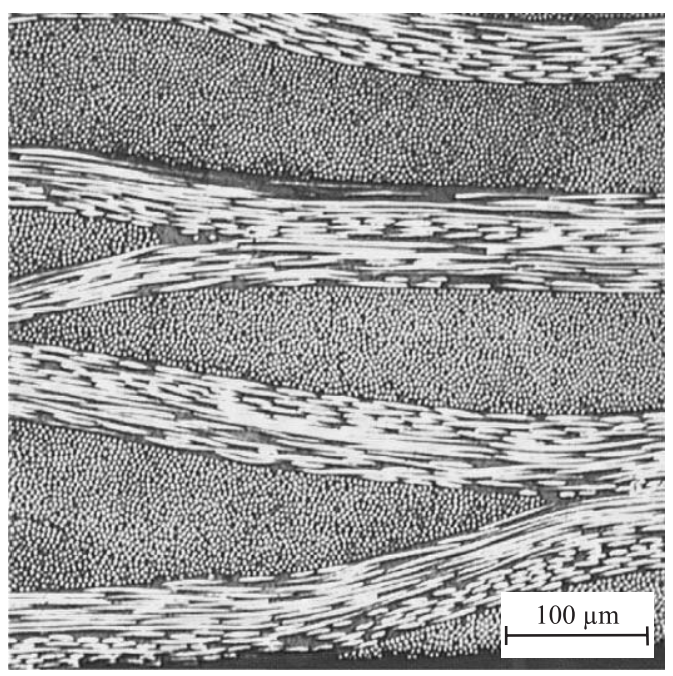

Figura 3. Micrografia óptica do compósito laminado de tecido de fibras de carbono/resina epóxi, representativa da qualidade de consolidação dos laminados processados. materiais para evitar delaminações e não danificar as propriedades mecânicas dos compósitos ${ }^{[10,11,15,16,26]}$.

\section{Análise microestrutural dos compósitos laminados base processados}

A micrografia mostrada na Figura 3 é representativa do padrão de qualidade obtido na compactação das camadas dos laminados base de tecido de fibras de carbono, vidro e aramida, curados em autoclave, antes da realização dos reparos. Os efeitos combinados de temperatura, pressão e vácuo foram fundamentais na obtenção dos laminados com espessura uniforme e isentos de defeitos, onde os espaços foram completamente preenchidos pela resina, como já havia sido assegurado pela inspeção ultra-sônica. Esta característica é atribuída à relação do fluxo da resina com o adequado tempo de gelificação (ou ponto de gel) e todos os parâmetros de moldagem controlados (patamares de temperatura, pressão, vácuo) ${ }^{[15,27]}$ durante a moldagem dos laminados.

\section{Análise térmica dinâmico-mecânica}

Inicialmente foram analisadas amostras sem reparos, constituídas de compósitos de tecidos de fibras de carbono com as resinas epóxi F155 e F584, de modo a determinar as temperaturas de transição vítrea $\left(\mathrm{T}_{\mathrm{g}}\right)$ destes sistemas utilizados como laminado base e reparo nos compósitos de fibras de carbono reparados. Na Figura 4 estão apresentadas as curvas dinâmico-mecânicas com o módulo de armazenamento $\left(E^{\prime}\right)$, módulo de perda $\left(E\right.$ ') e tan delta $\left(E{ }^{\prime \prime} / E^{\prime}\right)$ do compósito laminado de tecido de fibras de carbono com a resina epóxi F155. Neste trabalho, a determinação da temperatura de transição vítrea foi feita adotando-se o máximo do módulo de perda ( $E$ "), podendo-se verificar que a $\mathrm{T}_{\mathrm{g}}$ da resina $\mathrm{F} 155$ é igual a $126^{\circ} \mathrm{C}$.

Foi adotado o módulo de perda ( $E$ ") porque este apresenta um pico máximo em temperaturas um pouco menores do que o pico máximo de fricção interna (tan $\left.\delta=E^{\prime \prime} / E^{\prime}\right)$. O máximo de dissipação de calor por unidade de deformação ocorre na temperatura em que E" é máximo. Esta temperatura, a $1 \mathrm{~Hz}$ de freqüência, está mais próxima ao valor de temperatura de transição vítrea determinada por outros métodos, como DSC. Desta maneira, utilizou-se o módulo de perda E"

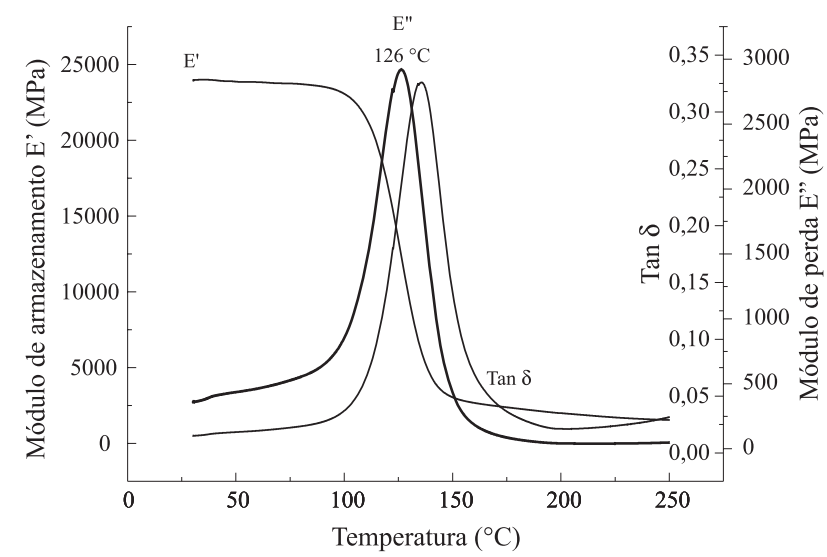

Figura 4. Curvas DMTA do laminado base de tecido de fibras de carbono com resina epóxi F155. 


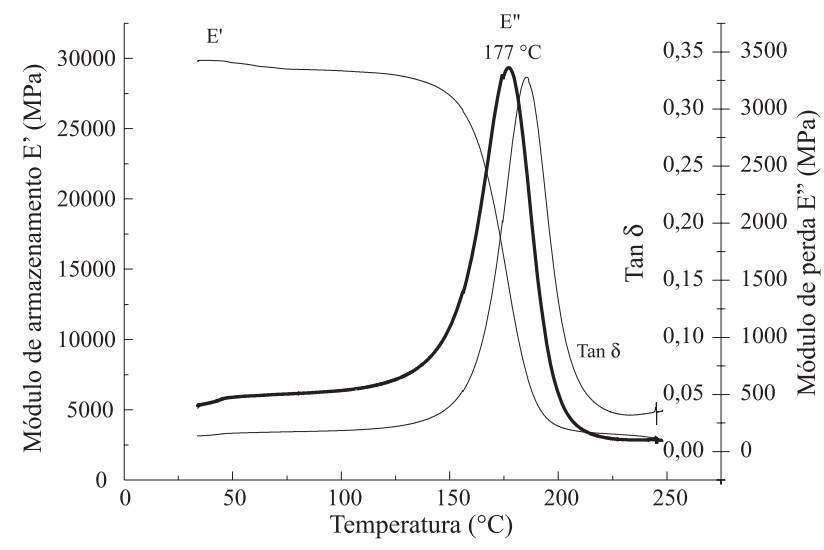

Figura 5. Curvas DMTA do laminado base de fibras de carbono com a resina epóxi F584.

Tabela 1. Temperatura de transição vítrea $\left(\mathrm{T}_{\mathrm{g}}\right)$ das resinas epóxi F155 e F584.

\begin{tabular}{ccc}
\hline Sistema de resinas & $\mathbf{T}_{\mathbf{g}}\left({ }^{\circ} \mathbf{C}\right)$ & $\mathbf{T}_{\mathbf{g}}\left({ }^{\circ} \mathbf{C}\right)$ determinada \\
\hline F155 & $121^{*}$ & 126 \\
F584 & $171^{*}$ & 177 \\
Filme adesivo & $95^{* *}$ & 93 \\
\hline
\end{tabular}

* Catálogo da Hexcel Composites ${ }^{[28]}$

** Catálogo da Cytecfiberite ${ }^{[29]}$

para se determinar a região de transição vítrea dos materiais poliméricos $^{[19]}$.

Na Figura 5 são mostradas as curvas DMTA do compósito laminado de tecido de fibras de carbono com a resina epóxi F584, que apresenta a sua temperatura de transição vítrea em $177^{\circ} \mathrm{C}$. As temperaturas de transição vítrea encontradas para os sistemas F155 e F584 estão próximas às citadas pelo fabricante dos pré-impregnados (Hexcel Composites), que determinou as temperaturas $T_{g}$ das resinas epóxi utilizadas (Tabela 1). As pequenas variações de temperatura $T_{g}$ observadas neste trabalho, em relação às do fabricante dos pré-impregnados, podem ser atribuídas às variações físico-químicas de diferentes lotes de uma mesma resina, ao ciclo térmico utilizado na cura da resina e/ou à técnica de determinação da $\mathrm{T}_{\mathrm{g}}{ }^{[19]}$, que no caso deste trabalho foi realizada diretamente em uma amostra na forma de pequena barra do respectivo compósito laminado.

Para comparação e informação, na Tabela 2 estão mostrados os valores de $\mathrm{T}_{\mathrm{g}}$ citados pelo fabricante dos pré-impregnados (Hexcel Composites) e os valores determinados por DMTA em trabalho anterior ${ }^{[30]}$, no qual foram estudadas as

Tabela 2. Temperatura de transição vítrea $\left(\mathrm{T}_{\mathrm{g}}\right)$ após condicionamento higrotérmico de compósitos contendo resinas epóxi F155 e F584.

\begin{tabular}{ccc}
\hline Sistema de resinas & $\mathbf{T}_{\mathbf{g}}\left({ }^{\circ} \mathbf{C}\right)$ & $\mathbf{T}_{\mathbf{g}}\left({ }^{\circ} \mathbf{C}\right)$ determinada \\
\hline F155 & $99 *$ & $112 * *$ \\
F584 & $121 *$ & $136 * *$ \\
\hline
\end{tabular}

* Catálogo da Hexcel Composites ${ }^{[28]}$

** Trabalho anterior ${ }^{[30]}$ alterações das $T_{g}$ de compósitos de matrizes de resinas epóxi F155 e F584 após condicionamento higrotérmico (temperatura e umidade elevadas).

Baseando-se nos valores da Tabela 2 e comparando-se com a Tabela 1 pode-se verificar que o condicionamento higrotérmico ocasiona um abaixamento da $\mathrm{T}_{\mathrm{g}}$ dos compósitos laminados contendo as respectivas resinas epóxi F155 e F584. A diminuição da $T_{g}$ dos compósitos está relacionada ao efeito plastificante que a água provoca entre as cadeias da rede polimérica da matriz epóxi ${ }^{[14,30,31,32]}$.

\section{DMTA dos compósitos laminados reparados}

\section{Laminados base de tecido de fibras de carbono repa- rados com pré-impregnado de tecido de fibras de car- bono}

Nas Figuras 6 a 14 estão apresentadas as curvas DMTA das amostras dos laminados base de fibras de carbono/resina F584 reparados com pré-impregnados de fibras de carbono com resina F155, retiradas de diferentes partes do corpo-de-prova de tração já ensaiado. Analisando-se as Figuras 6 e 7 observa-se a presença bem definida de dois picos de $E$ ” e dois de tangente

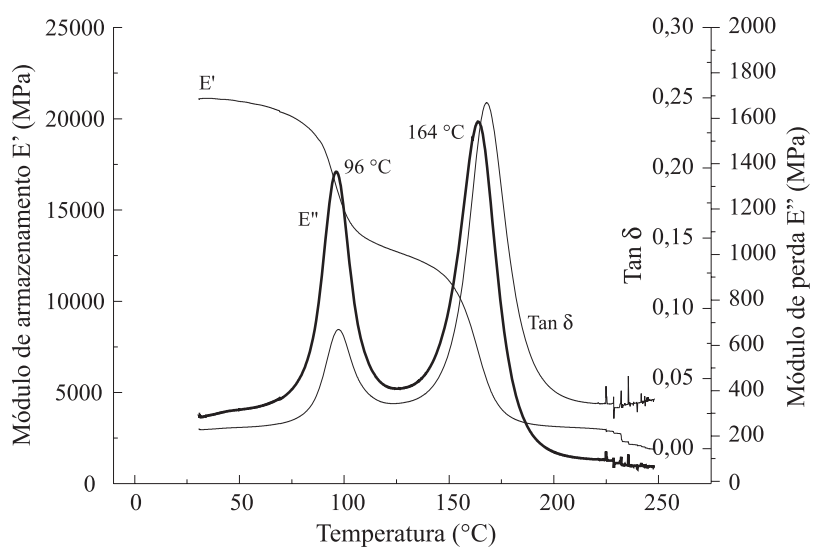

Figura 6. Curvas DMTA do compósito laminado de tecido de fibras de carbono/epóxi F584 reparado com tecido de fibras de carbono/epóxi F155. Amostra retirada da região próxima à falha do corpo-de-prova ensaiado em tração.

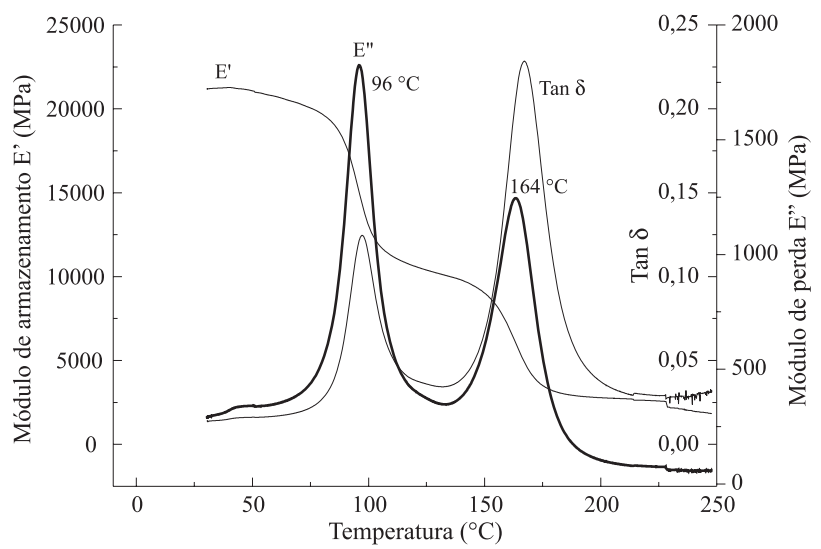

Figura 7. Curvas DMTA do compósito laminado de fibra de carbono/F584 reparado com fibra de carbono/F155. Amostra retirada da região próxima ao tab do corpo-de-prova ensaiado em tração. 
delta $(\tan \delta)$, que são referentes às contribuições dos dois sistemas de resinas. $\mathrm{O}$ primeiro pico refere-se à contribuição do sistema de resina F155 utilizado no reparo e o segundo ao sistema de resina F584 utilizado na manufatura do laminado base. Observa-se, ainda, que as $T_{g}$ dos dois sistemas de resina, quando atuando em conjunto diminuíram $30^{\circ} \mathrm{C}$ para a resina epóxi F155 e $13{ }^{\circ} \mathrm{C}$ para a F584, diminuindo, conseqüentemente, a temperatura de serviço do laminado.

Comparando-se as Figuras 6 e 7 com as obtidas para os laminados base isolados (Figuras 4 e 5) observa-se que, os módulos de armazenamento (E') diminuíram para cerca de $20.000 \mathrm{MPa}$ (na região de temperatura de 25 a $50^{\circ} \mathrm{C}$ ), ou seja, aproximam-se mais do compósito com epóxi F155, a qual é a resina utilizada no reparo. Sugere-se que tal decréscimo também deve ter sido influenciado pela presença do filme adesivo epóxi utilizado na execução do reparo dos laminados base, porque este filme não é um material estrutural com propriedades mecânicas elevadas, trata-se de um material constituído basicamente de resina epóxi, e que apresenta somente uma tela interna para suportar o adesivo. Neste ponto, é preciso ressaltar que é necessária a utilização de filme adesivo no reparo das peças aeronáuticas porque este melhora a adesão na interface reparo/laminado base curado.

A Figura 8 é representativa da região central do corpo-deprova de tração, ou seja, da região de reparo, sendo constituída essencialmente do compósito laminado de reparo, que foi realizado com pré-impregnado de tecido de fibras de carbono com a resina F155. Neste caso, é observada somente a contribuição do sistema de resina epóxi F155, porém a curva referente ao módulo de perda (E") mostra um alargamento e uma pequena alteração (ombro) à esquerda (em torno da temperatura de $93{ }^{\circ} \mathrm{C}$ ), proveniente da contribuição do filme adesivo (revelando uma cura incompleta) posicionado entre o laminado base e o material de reparo. Novamente, observase o abaixamento da $T_{g}$ do sistema epóxi F155, ficando mais evidente na análise desta figura a contribuição da $T_{g}$ do filme adesivo no abaixamento deste parâmetro para o sistema de resina do reparo.

Com o objetivo de se comprovar a contribuição do filme adesivo epóxi e verificar a $T_{g}$ do filme isoladamente, foi reali-

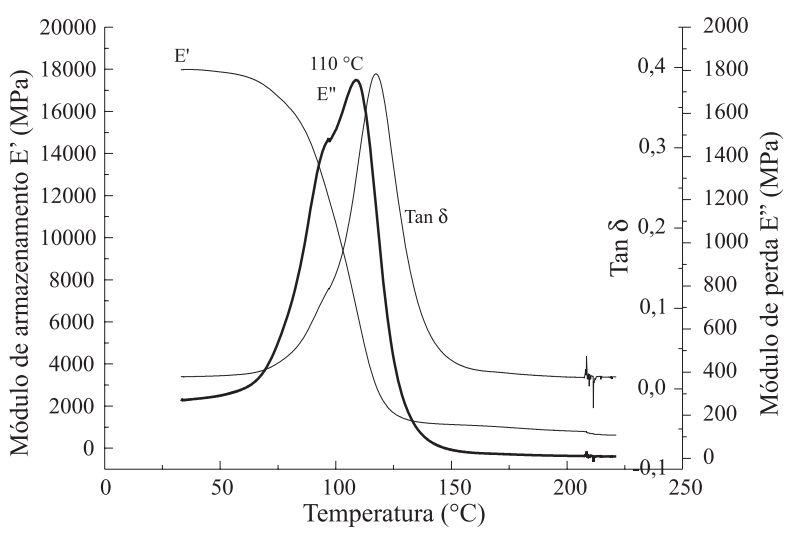

Figura 8. Curvas DMTA do compósito laminado de fibra de carbono/F584 reparado com fibra de carbono/F155. Amostra retirada da região central do corpo-de-prova ensaiado em tração.

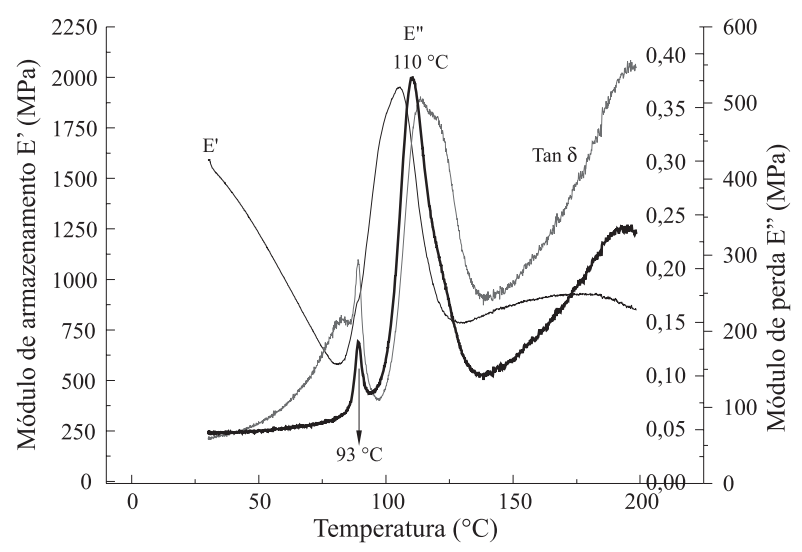

Figura 9. Curvas DMTA do filme adesivo epóxi, submetido ao mesmo ciclo de cura dos laminados reparados.

zada a cura deste adesivo em estufa, submetendo-o ao mesmo ciclo de cura dos laminados reparados e, posteriormente, foi realizada a análise do filme adesivo por DMTA (Figura 9).

A análise da Figura 9 revela que o filme submetido ao ciclo de cura utilizado no reparo apresenta duas alterações na curva $E$ ” relacionadas à $T_{g}$ do material, sendo uma a cerca de $93^{\circ} \mathrm{C}$ e outra a $110^{\circ} \mathrm{C}$. De acordo com o fabricante do filme adesivo a $T_{g}$ seria $95{ }^{\circ} \mathrm{C}$ (Tabela 1 ) e a temperatura de serviço $84^{\circ} \mathrm{C}^{[29]}$.

Assim, analisando-se novamente a Figura 8 pode se verificar que na curva de DMTA, correspondente à região central do corpo-de-prova analisado, aparecem características da $T_{g}$ do filme adesivo de epóxi utilizado no reparo do laminado, contribuindo, assim, para o abaixamento da $T_{g}$ do sistema de reparo.

Na Figura 9 pode-se verificar, também, que o filme epóxi durante a varredura no DMTA apresenta decréscimo no módulo de armazenamento $E^{\prime}$ até próximo a $90{ }^{\circ} \mathrm{C}$ e, em seguida, o aumento do módulo devido à varredura da temperatura. $\mathrm{O}$ aumento do módulo está relacionado ao prosseguimento da reação de cura (reticulação da resina epóxi) em algumas regiões do material adesivo. Logo após é observada a movimentação de partes mais livres da cadeia, indicando a $T_{g}$ a $110^{\circ} \mathrm{C}$. Na realidade, no intervalo de temperatura da $T_{g}$, o polímero passa a apresentar movimentos difusionais envolvendo movimentos rotacionais de segmentos da cadeia e translacionais dos finais da cadeia, mas não envolvendo a movimentação das moléculas como um todo. Este fenômeno provoca a diminuição no módulo de armazenamento $(E$ ') em cerca de 3 a 4 ordens de grandeza, e o aumento do módulo de

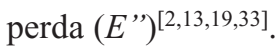

Na Figura 10 estão apresentadas as curvas DMTA do filme adesivo epóxi após este ser submetido a dois ciclos de cura, pois suspeitava-se que o mesmo não estava adequadamente curado. Desta maneira, na figura ainda pode ser observado o aumento no módulo $E$ ' e a transição vítrea a $110^{\circ} \mathrm{C}$, no entanto, já não é mais observada a transição em torno de $93^{\circ} \mathrm{C}$, devido a cura agora ter sido realizada adequadamente.

Em resumo, a análise das curvas DMTA dos laminados base de carbono reparados, sem prévio condicionamento higrotérmico, mostra os efeitos da combinação de diferentes 


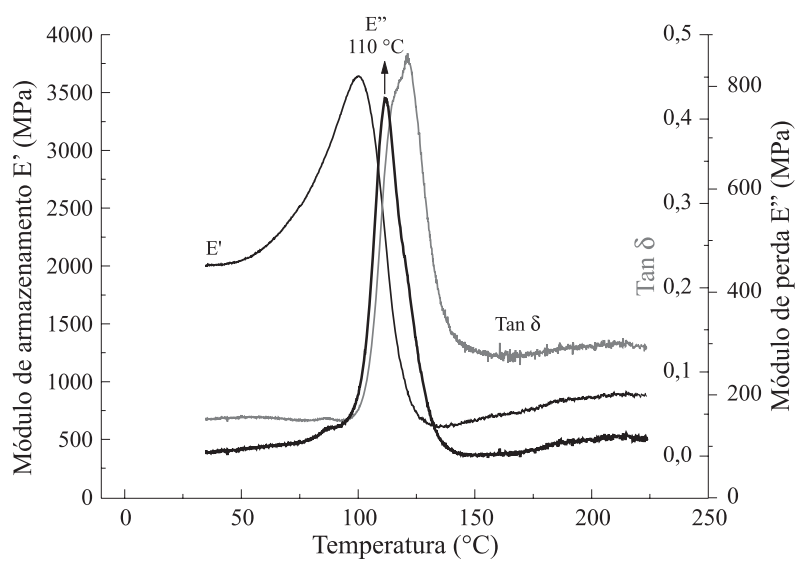

Figura 10. Curvas DMTA do filme adesivo epóxi, submetido a dois ciclos de cura.

sistemas de resinas nas suas respectivas temperaturas de transição vítrea e, conseqüentemente, na temperatura de serviço do componente reparado. Pelos resultados apresentados até o momento verifica-se que a combinação de sistemas de resina epóxi de diferentes naturezas e com diferentes $T_{g}$, formando uma interface com um filme adesivo de $T_{g}$ mais baixa, promove o abaixamento das temperaturas de transição vítrea de todo o sistema de laminado base reparado. Esta é uma informação importante e que deve ser levada em consideração na aplicação destes reparos estruturais na área aeronáuti$\mathrm{ca}$, isto porque a temperatura de serviço do componente reparado precisa ser inferior à $T_{g}$.

Além disto, pode-se verificar a necessidade de dois ciclos de cura (ou aumento no tempo de cura) nos laminados reparados com os filmes adesivos de epóxi, pois desta forma não se observará alteração do comportamento dinâmico-mecânico dos laminados na temperatura de aproximadamente $93{ }^{\circ} \mathrm{C}$.

\section{Laminados base de tecido de fibras de vidro reparados com tecido de fibras de vidro}

Nas Figuras 11 e 12 estão apresentados os comportamentos dinâmico-mecânicos dos laminados base de tecido de fibras de vidro reparados com tecido de fibras de vidro.

A Figura 11 mostra que a $T_{g}$ determinada está próxima dos valores observados para os compósitos laminados de carbono, os quais foram reparados com o mesmo tipo de filme adesivo de epóxi. Também, neste caso, pode ser observada a influência da $T_{g}$ do filme adesivo no abaixamento da $T_{g}$ do sistema de resina do reparo. Este aspecto observado é atribuído ao fato do corpo-de-prova analisado, apesar de ter sido retirado mais próximo do $t a b$, apresenta-se com uma fase representativa do adesivo. Este comportamento, levando ao abaixamento da $T_{g}$, está relacionado ao fato da migração da resina epóxi (do filme adesivo, que contém oligômeros) durante o processo de cura, favorecendo a interpenetração de cadeias. Visualmente, também, verificou-se a migração da resina epóxi do filme adesivo devido à coloração azul deste, principalmente, quando observou-se o corpo-de-prova do laminado contendo fibra de vidro, pois este apresentava anteriormente coloração amarela clara.

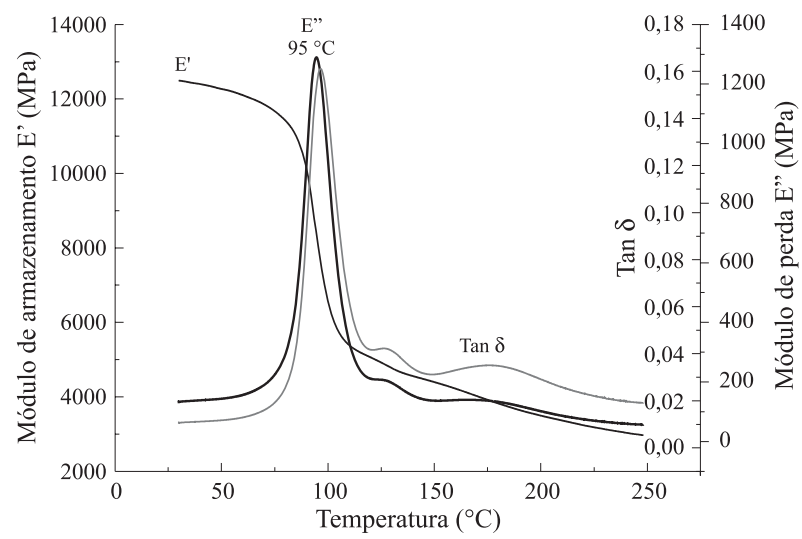

Figura 11. Curvas DMTA do compósito de fibra de vidro/epóxi F161, reparado com fibra de vidro/epóxi F155. Amostra retirada de região próxima ao tab do corpo-de-prova ensaiado em tração.

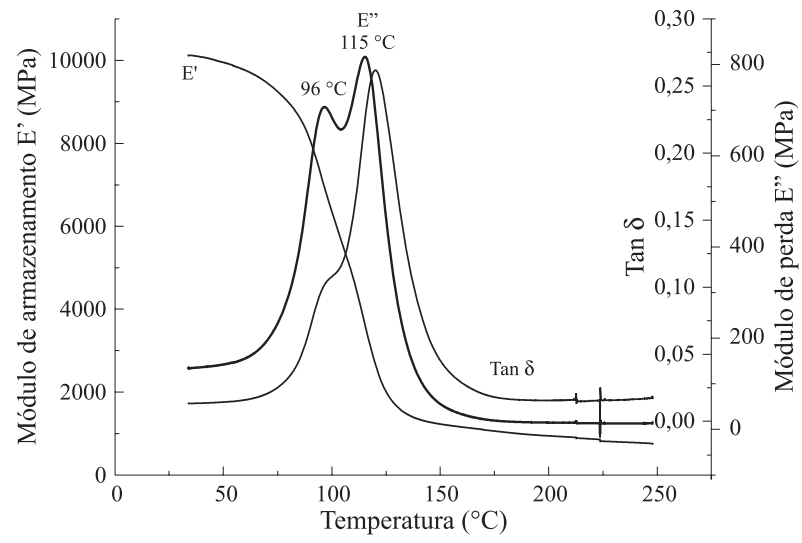

Figura 12. Curvas DMTA do compósito de fibra de vidro/epóxi F161 reparado com fibra de vidro/epóxi F155. Amostra retirada de região central do corpo-de-prova ensaiado em tração.

Na Figura 11 podem ser observadas, ainda, alterações nas curvas de módulo de perda ( $E$ ") e tan delta, que estão relacionadas à presença da resina $\mathrm{F} 155\left(T_{g}\right.$ próxima a $\left.126^{\circ} \mathrm{C}\right)$ utilizada no reparo e à presença da F161 ( $T_{g}$ próxima a $180^{\circ} \mathrm{C}$ ) relacionada ao laminado base de tecido de fibras de vidro. Estes dois tipos de resinas epóxi (provenientes de $D G E B A$ ) apresentam estruturas químicas similares e também pode ocorrer a interpenetração de cadeias durante o processo de cura.

Na Figura 12 são observadas alterações nos módulos de perda $\left(E^{\prime \prime}\right)$ relacionadas à $T_{g}$ do filme adesivo $\left(96^{\circ} \mathrm{C}\right)$, o qual contribui para o abaixamento da $T_{g}$ da resina $\mathrm{F} 155$ para $115^{\circ} \mathrm{C}$. Nesta amostra que foi retirada da região central do corpo-de-prova não são observadas características do laminado base (resina F161), o qual não está presente ou está em pequena quantidade, revelando que nesta região predominam as propriedades do filme adesivo e da resina de reparo.

\section{Laminados base de aramida reparados com fibras de aramida}

Nas Figuras 13 e 14 estão apresentadas as curvas DMTA dos laminados que foram submetidos à condição mais crítica, ou seja, foram climatizados (temperatura e umidade elevadas) para o ensaio de tração realizado anteriormente. Assim, 


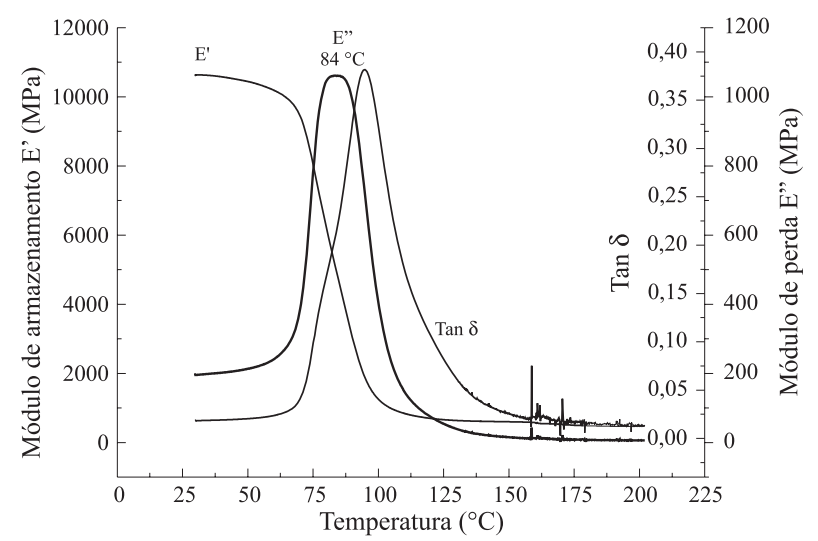

Figura 13. Curvas de DMTA do compósito de fibra de aramida/F161 reparado com fibra de aramida/F155. Amostra retirada de região próxima ao $t a b$ do corpo-de-prova ensaiado em tração.

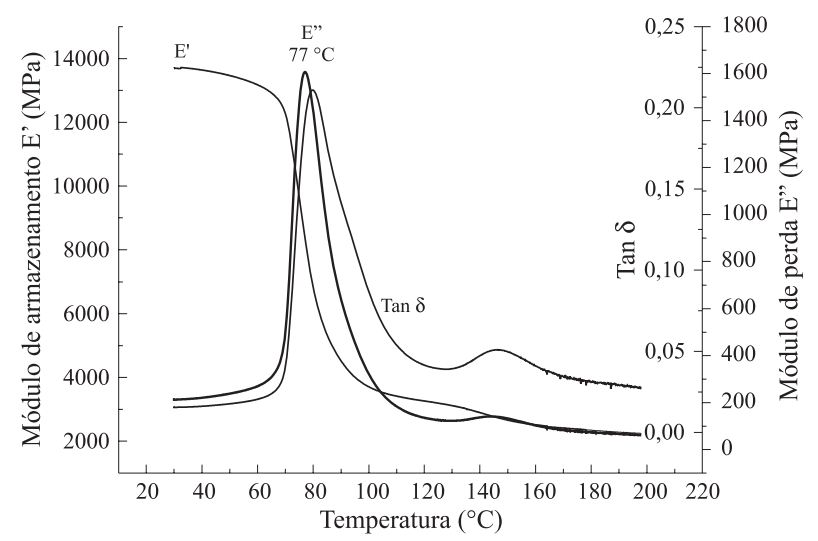

Figura 14. Curvas DMTA do compósito de tecido de fibras de aramida/ epóxi F161 reparado com tecido de fibras de aramida/epóxi F155. Amostra retirada de região central do corpo-de-prova ensaiado em tração.

as Figuras 13 e 14 mostram uma redução mais acentuada da temperatura de transição vítrea, que o observado anteriormente para os laminados base de tecido de fibras de carbono (Tabela 2). Os valores de $T_{g}$ dos laminados reparados de tecido de fibras de aramida revelam que as moléculas de água ocasionaram um efeito plastificante intenso sobre o sistema de resina epóxi (F155) do reparo combinado com o filme adesivo de epóxi, a resina epóxi F161, e ainda as fibras de aramida, provocando uma acentuada redução da $T_{g}$. Portanto, a presença de água (umidade) e a exposição à temperatura elevada deste sistema de resinas epóxi ocasionam a formação de um "sistema modificado", com o abaixamento da $T_{g}$ e, conseqüentemente, da respectiva temperatura de serviço do laminado. Estes resultados explicam os dados obtidos anteriormente em ensaios em tração ${ }^{[10,11]}$, na condição higrotérmica (combinação de temperatura e umidade elevadas), quando todos os corpos-de-prova falharam na região do adesivo por cisalhamento da interface reparo/laminado base, recompondo, em média, somente $10 \%$ das propriedades mecânicas deste laminado, nesta condição de ensaio. Estes resultados estavam muito abaixo dos obtidos quando os corpos-de-prova foram testados em condições ambiente úmida (acondicionados em umidade relativa em torno de 95\%, e temperatura de $60^{\circ} \mathrm{C}$ até a saturação).
Como observado neste caso, a exposição higrotérmica por um período de longa duração provoca efeitos irreversíveis, devido à interação da água com grupos funcionais de matrizes poliméricas de natureza polar, como os grupos presentes na resina epóxi. Este fenômeno provoca a diminuição das interações físico-químicas existentes entre resina e fibra e levam à degradação da interface reforço/matriz polimérica, conduzindo à deterioração das propriedades mecânicas dos compósitos poliméricos ${ }^{[14,31,32,34]}$.

\section{Conclusões}

A análise das curvas DMTA dos laminados base de tecido de fibras de carbono reparados mostrou os efeitos da combinação de diferentes sistemas de resinas na temperatura de transição vítrea e, conseqüentemente, na temperatura de serviço do componente reparado. Pelos resultados apresentados até o momento verificou-se que a combinação de sistemas de resina epóxi de diferentes naturezas (F584 e F155) e com diferentes $T_{g}$, formando uma interface com um filme adesivo de epóxi de $T_{g}$ mais baixa, ocasionou o abaixamento das temperaturas de transição vítrea de todo o sistema do laminado base reparado. No caso dos laminados de vidro reparados, contendo resinas F161 e F155, observou-se com evidência (indicada também pela coloração) a migração do filme adesivo de epóxi para regiões do laminado de reparo. Todas estas informações são importantes e devem ser levadas em consideração na aplicação destes reparos estruturais.

Portanto, com a verificação da temperatura de transição vítrea $\left(T_{g}\right)$ por DMTA determinou-se a causa pela qual vários laminados reparados não apresentaram as propriedades mecânicas previstas, mesmo após sendo verificado que não havia defeitos (bolhas, vazios, delaminações, etc.) nestes materiais.

Verificou-se pela análise das curvas DMTA que os laminados reparados de tecido de fibras de aramida/epóxi são bastante susceptíveis à umidade e temperatura elevadas, ocasionando uma baixa temperatura de transição vítrea.

Assim, conclui-se que a submissão dos compósitos laminados reparados de fibras de aramida à temperatura e umidade elevadas degrada a adesão na interface formada na colagem e, conseqüentemente, as propriedades mecânicas e térmicas do adesivo epóxi utilizado no reparo. Portanto, para estes sistemas de resina combinados com tecidos de fibras de aramida utilizados não é recomendada a exposição dos respectivos laminados reparados (ou peças reparadas) às condições higrotérmicas consideradas neste trabalho.

\section{Agradecimentos}

À FAPESP (Processo 02/02057-5), ao CNPq (Processo 303528/2003-6), e às empresas EMBRAER pelo fornecimento dos pré-impregnados e $d p U N I O N$ pelo empréstimo do equipamento DMA.

\section{Referências Bibliográficas}

1. Baker, A. A.; Callus, P. J.; Georgiadis, S.; Falzon, P. J.; 
Dutton, S. E.; Leong, K. H. - Composites: Part A, 33, p. 687-696 (2002).

2. Hayes, B.S.; Gilbert, E.N.; Seferis, J.C. - Composites Part A: Appl. Sci. and Manufacturing, 31, p. 717-725 (2000).

3. Penn, L. S.; Wang, H. Epoxy Resins, in: Handbook of Composites. 2 ed., S. T. Peters (ed), Chapman \& Hall, Great Britain (1998).

4. Pilato, L.A.; Michno, M.J. - "Advanced Composite Materials", Springer Verlag Berlin Heidelberg, Berlin, Germany (1994).

5. Shim, S.B.; Seferis, J.C.; Eom, Y.S.; Shim, Y.T. Thermochimica Acta, 291, p.73-79 (1997).

6. Harper, C. A. - "Handbook of Plastics, Elastomers and Composites". 3. ed., McGraw-Hill Companies, USA (1996).

7. Seymour, R.B. - "Polymeric Composites, New Concepts in Polymer Science". CRHI, The Netherlands (1990).

8. Schwartz, M. M. - SAMPE Journal, 32, p.18 (1996).

9. Schwartz, M. M. - "Composite Materials. Properties, Nondestructive Testing and Repair", Prentice Hall Inc, New Jersey, USA, v.1 (1997).

10. Mayer, S. "Influência do Condicionamento Ambiental na Resistência à Tração de Laminados de Compósitos Poliméricos Reparados". Dissertação de Mestrado.Instituto Tecnológico de Aeronáutica, ITA, Brasil (2003).

11. Mayer, S.; Cândido, G. M.; Rezende, M. C. - Polímeros: Ciência e Tecnologia, 13, n.3, p.147-153 (2003).

12. Wu, K.-W.; Lee, C.-L., Chang Y.-C.; Ong, C-L. - Materials Chemistry and Physics, 43, p. 173-177 (1996).

13. Wingard, C.D. Thermochimica Acta, 357-358, p.293-301 (2000).

14. Choi, H. S.; Ahn, K. J.; Nam, J.-D.; Chun, H. J. Composites: Part A, 32, p. 709-720 (2001).

15. Cândido, G.M.; Almeida, S. F. M.; Rezende, M. C. Polímeros: Ciência e Tecnologia, 10, n.1, p. 31-41 (2000).

16. Abaris Training - "Preliminary Design of Composites Structures". Reno NV, EUA, (2000).

17. Courter, J. L. - "Thermal Analysis", in: Composites. Engineered Materials Handbook, Metals Park, section 11, OH: ASM., v.1 (1987).
18. Handbook MIL. - "Guidelines for Property Testing of Composites". MIL-HDBK-17-1F, v.1 (2002).

19. Murayama, T. - "Dynamic Mechanical Analysis of Polymeric Material”, Elsevier Scientific Publishing Company, Amsterdam (1982).

20. Tanoglu, M.; Robert, S.; Heider, D.; McKnigth, S.H.; Brachos, V.; Gillespie Jr., J.W. - International Journal of Adhesion \& Adhesives, 21, p.187-195 (2001).

21. Keusch, S.; Haessler, R. - Composites Part A Applied Science and Manufacturing, 30, p. 997-1002 (1999).

22. Canevarolo Jr., S. V. - "Análise Dinâmico-Mecânica para Sistemas Poliméricos", in: Revista da ABPol, p.36-40 (1991).

23. Menard, K. P. - "Dynamic Mechanical Analysis: A Practical Introduction", CRC Press LLC, USA (1999).

24. Wetton, R. E.; Marsh, R.D.L.; Van-de-Velde, J.G. Thermochimica Acta, 175, p.1-11 (1991).

25. Ray, D.; Sarkar, B.K.; Das, S.; Rana, A. K. Composites Science and Technology, 62, p.911-917 (2002).

26. Abaris Training. "Advanced Composite Structures: Fabrication and Damage Repair", Reno NV, EUA (1998).

27. Costa, M. L.; Paiva, J. M. F.; Botelho, E. C.; Rezende, M. C. - Polímeros: Ciência e Tecnologia, 3, p.188-197 (2003).

28. Hexcel Composites. - "Resin systems for advanced composites" in: Catalog of Hexcel Composites, Pleasanton (2000).

29. Cytecfiberite. "Catalog of Cytecfiberite", East Technology Circle Tempe, Arizona (1998).

30. Paiva, J. M. F.; Costa, M. L.; Rezende, M. C. - PolymerPlastics Technology and Engineering, 45, p.1-8 (2006).

31. Vanlandingham, M. R.; Eduljee, R. F.; Gillespie, J. W., Jr. - Journal of Applied Polymer Science, 71, p. 787-798 (1999).

32. Wollf, E. G. - SAMPE Journal, 29, n.3, p.11-19 (1993).

33. Hatakeyama, T. Quinn, F.X. "Thermal Analysis Fundamentals and Applications to Polymer Science", John Wiley \& Sons, England (1994).

34. Wang, J.-Y.; Ploehn, H. J. - "Journal of Applied Polymer Science", 59, p.345-357 (1996).

Enviado: 12/05/05

Reenviado: 03/02/06

Aprovado: 07/02/06 